Intersections

Canadian Journal of Music

Revue canadienne de musique
Intersections CANADAN TOURAA OP NUSIC

\title{
Didier van Moere. 2008. Karol Szymanowski. Paris : Fayard. 695 p. ISBN 978-2-213-63774-7 (couverture cartonnée)
}

\section{Justine Comtois}

Volume 28, numéro 2, 2008

URI : https://id.erudit.org/iderudit/029958ar

DOI : https://doi.org/10.7202/029958ar

Aller au sommaire du numéro

\section{Éditeur(s)}

Canadian University Music Society / Société de musique des universités canadiennes

ISSN

1911-0146 (imprimé)

1918-512X (numérique)

Découvrir la revue

Citer ce compte rendu

Comtois, J. (2008). Compte rendu de [Didier van Moere. 2008. Karol Szymanowski. Paris : Fayard. 695 p. ISBN 978-2-213-63774-7 (couverture cartonnée)]. Intersections, 28(2), 104-109. https://doi.org/10.7202/029958ar

Tous droits réservés (C) Canadian University Music Society / Société de musique des universités canadiennes, 2009
Ce document est protégé par la loi sur le droit d'auteur. L’utilisation des services d'Érudit (y compris la reproduction) est assujettie à sa politique d'utilisation que vous pouvez consulter en ligne.

https://apropos.erudit.org/fr/usagers/politique-dutilisation/ 
Toffler, Alvin, 2007. La richesse révolutionnaire. Paris : Plon.

Théberge, Paul. 1997. Any Sound You Can Imagine: Making Music/Consuming Technology. Middletown : Wesleyan University Press.

Wittgenstein, L. J. 1953. Philosophische Untersuchungen-Philosophical Investigations. Oxford : Blackwell.

JESSICA RoDA

Didier van Moere. 2008. Karol Szymanowski. Paris : Fayard. 695 p. ISBN 978-2213-63774-7 (couverture cartonnée).

"J'aime ce qui est délaissé ». C'est en commençant cette biographie par une citation de Vladimir Jankélévitch (tirée d'un entretien radiophonique) que Didier van Moere rappelle à notre mémoire (p. 9) l'importance de ce compositeur polonais (1882-1937). Ce livre, qui vient tout juste de paraître dans la collection des éditions Fayard destinée aux biographies de musiciens, offre un panorama très complet de la vie, de l'œuvre et de la pensée de cet héritier de Chopin. Il s'agit ici d'un ouvrage de référence indispensable pour quiconque s'intéresse à ce compositeur, ou encore, de manière plus générale, à la musique polonaise du début du $\mathrm{XX}^{\mathrm{e}}$ siècle, ou même aux musiques étrangères présentes à Paris à cette époque. Rappelons à cet effet que Szymanowski a participé de façon très active au milieu musical français, comme le mentionne Michel Duchesneau, notamment en s'associant régulièrement aux activités de la SMI : « Milhaud ne cesse de réclamer que l'on joue les œuvres de Szymanowski, car il voit en ce compositeur un représentant de tout premier ordre de la musique moderne » (1997, p. 160).

Un ouvrage exhaustif portant sur ce compositeur manquait grandement à la littérature musicologique. Nous n'avions jusqu'à présent que de rares biographies, malheureusement incomplètes et qui remontent aux années 1990 (notamment celles de Christopher Palmer (1987), et de Jim Samson (1990)).

Agrégé de lettres classiques et docteur en musicologie, Didier van Moere, auteur d'une thèse sur Szymanowski et la France, soutenue à l'Université Paris Sorbonne (Paris IV) en 1993, enseigne maintenant les rapports entre la littérature et la musique à l'Université Stendhal-Grenoble 3 en France. Il a entrepris, pour la publication de ce livre, le dépouillement de fonds d'archives gigantesques, tels ceux de la bibliothèque universitaire de Varsovie, la correspondance générale de Szymanowski et l'ensemble de ses écrits, musicaux et littéraires.

La vie de Szymanowski est présentée dans l'ordre chronologique. La présentation acquiert un grand intérêt du fait que les éléments biographiques sont entrecoupés par les analyses musicales, génétiques et stylistiques des œuvres de Szymanowski. Ces descriptions des œuvres sont présentées en retrait, dans le texte, et dans une police différente, ce qui rend la lecture très aisée.

À travers les onze chapitres qui constituent le livre, van Moere trace un portrait très utile de la situation sociopolitique de l'époque que traverse le protagoniste. 
Les deux premiers chapitres sont consacrés à l'enfance et aux années d'apprentissage de Szymanowski, ainsi qu'à ses premières compositions. Le jeune polonais, né à Tymószówka près de Kiev en 1882, parfait son éducation musicale et pianistique auprès de Gustav Neuhaus (1853-1937), qui dirige une école de musique qu'il a fondée à Ielisavetgrad (Ukraine). On apprend ainsi que Szymanowski est le cousin du grand pianiste polonais Heinrich (Harry) Neuhaus (1888-1964), fils de Gustav.

Les Neuf Préludes pour piano op. 1 (1899-1900) dénotent l'influence quasi inévitable de Chopin sur Szymanowski. Cependant, «alors qu'il aurait pu opter pour des mazurkas ou des polonaises, [Szymanowski] choisit le Chopin le plus classique et le moins polonais » (p. 30). Van Moere attribue cette caractéristique à la modestie du compositeur qui, contrairement à Scriabine ou Bartók, opte davantage pour le "romantisme exténué que pour le romantisme flamboyant » (p. 31). Ces préludes pour piano incarnent le renouveau de la musique en Pologne, malgré qu'il s'agisse d'œuvres de jeunesse, qui ne montrent pas encore la pleine maturité de leur créateur.

À l'automne 1901, Szymanowski arrive à Varsovie, où il poursuit sa formation de compositeur auprès de Zygmunt Noskowski (1846-1909).

La rencontre du jeune violoniste Pawel Kochanski (1887-1934) sera très marquante pour le compositeur. "Quand le compositeur écrira pour le violon, ce sera pour lui et avec lui » (p. 57-58). Quelques années plus tard, en 1904, la rencontre d'Arthur Rubinstein (1887-1982) aura elle aussi un grand impact sur la carrière du compositeur. Ces trois jeunes musiciens formeront, comme l'explique le biographe, « un trio inséparable de frères en musique » (p. 74).

Le troisième chapitre est consacré à la Jeune Pologne en musique. Ce groupe aussi appelé Société d'édition des jeunes compositeurs polonais est patronné, grâce à l'instance de Grzegorz Fitelberg (1879-1953), par le prince Wladyslaw Lubomirski (1866-1934). La mission de cette société est de " prouver au public comme à la critique l'existence de la jeune musique polonaise » (p. 80). Le premier concert officiel de la société a lieu à la Philharmonie de Varsovie en 1906. C'est la première fois qu'on observe, comme le démontre van Moere, la volonté de créer une véritable école polonaise. Lors de ce concert, Szymanowski fait l'unanimité auprès du public et de la critique pour laquelle cet événement, d'après l'auteur, "ne passe pas inaperçu, chacun sentant qu'une renaissance de la musique polonaise permettrait de relever la tête face aux compositeurs si prestigieux des puissances occupantes » (p. 88-89).

Dans les chapitres IV et V, l'auteur s'attarde à la passion qui anime Szymanowski pour l'Italie et la culture grecque antique, qu'il découvre lors de son premier séjour en Calabre en $1911^{1}$. C'est à la même époque que le compositeur tente de percer une Vienne placée sous le signe de la modernité combative de Schoenberg et ses acolytes. Szymanowski participe aux nombreuses activités musicales organisées dans la capitale autrichienne ainsi quaux activités mondaines de la ville. C'est aussi durant cette période que notre musicien polonais pense à

1 Szymanowski témoigne d'une prédilection pour la littérature grecque, dont le Banquet de Platon qu'il apprécie tout particulièrement. 
se faire connaître à Paris. Cela aurait peut-être été possible avec l'aide de son ami Charles Cuvillier (1887-1955), rencontré à Vienne. Cependant un rendez-vous manqué avec Ravel, qui avait demandé à voir quelques-unes de ses mélodies, reportera l'expérience parisienne à plus tard. Et comme nous le rappelle le biographe, c'est en 1912 que Szymanowski s'emploie à l'instrumentation de son opéra Hagith op. 25, qui « se réfère à un épisode de la fin de la vie du roi David » (p. 166). Pour cette œuvre, le jeune compositeur polonais dit s'inquiéter, dans une lettre à Fitelberg (1912), de la trop forte influence de Richard Stauss, que l'on retrouvait déjà très présente dans l'Ouverture op. 12 ou dans la Deuxième Symphonie op. 19. Didier van Moere démontre cette influence en précisant que Rubinstein, ayant des réserves concernant cette partition, " aurait dissuadé Szymanowski de confier le rôle du Jeune Roi à un soprano travesti, ce qui eût été un emprunt incongru au Chevalier à la rose » (p. 169-170).

En 1915, Szymanowski complète ses Mythes op. 30, qui sont trois poèmes pour violon et piano ${ }^{2}$. C'est la toute première fois qu'il s'adonne à la musique à programme et rompt avec la tradition musicale. En effet, van Moere, dans une analyse exhaustive de l'œuvre précise que "Mythes est donc la première 'Nuit d'été' de Szymanowski qui, adhérant à l'esthétique impressionniste, conçoit son œuvre comme une évocation suggestive plus que comme une description. Le programme, aussi vague soit-il, est bien là, implicitement présent » (p. 190).

Le sixième chapitre aborde l'œuvre littéraire Ephebos, créée suite à une rencontre, en 1919, avec le jeune écrivain Boris Kochno (1904-1990), pour lequel Szymanowski aura un véritable coup de foudre. Il s'agit là du seul roman écrit par le compositeur. Seuls quelques passages cités dans la biographie demeurent, le reste du manuscrit ayant été détruit dans un incendie en septembre 1939. Ephebos contient quatre poèmes ${ }^{3}$ en langue française dans lesquels l'auteur décèle les influences très marquées de Baudelaire et Verlaine. Parce qu'il y déclare ouvertement son homosexualité, Szymanowski refusera de faire publier ses poèmes jusqu'à la mort de sa mère. Il les publiera sous le pseudonyme de Jan Wladyslaw Kostryn et «le dédie à tous ceux, 'connus ou inconnus,' qui ont souffert et qu'on a méprisés, victimes de la haine de l'opinion publique pour avoir compris que l'amour était plus fort que la mort» (p. 271) ${ }^{4}$.

Dans les septième et huitième chapitres, nous nous tournons vers les années 1920. C'est la conquête de l'Ouest de Szymanowski. Il participe régulièrement aux activités de l'association France-Pologne et, à Paris, Henry Prunières (18811942), directeur de La Revue musicale, le repère rapidement et lui voue une amitié indéfectible et un soutien sans faille.

Lors d'un séjour à New York en 1921, le compositeur polonais établit de nombreuses relations, notamment parmi les amis de Rubinstein, et rencontre Prokofiev, Rachmaninov et Lazare Saminski. Mais le musicien polonais n'envisage pas de carrière américaine. Sa place est définitivement en France, où il est régulièrement joué et où il trouve, contrairement aux États-Unis, l'appui de nombreux amis, notamment Henry Prunières.

2 La fontaine d'Aréthuse, Narcisse, Dryades et Pan.

3 " Ganymède. Little boy ", " Baedeker ", " N'importe ", "Vagabond ».

4 Cité par l'auteur et tiré de Ephebos. 
Szymanowski est de retour à Paris en 1921 et y rencontre Diaghilev et Stravinski. Rubinstein lui présente également le Groupe des Six, mais le seul qui montre quelque intérêt pour ses œuvres est Honegger. Lannée suivante, lors d'un des mardis musicaux organisés au Théâtre du Vieux-Colombier par La Revue musicale, Émile Vuillermoz définit Szymanowski comme le principal héritier de Chopin. L'auteur précise que « le critique pose Szymanowski en symbole d'une modernité rassurante, en anti-Milhaud surtout, dont le Cinquième Quatuor, dédié à Schoenberg et créé un mois plus tôt au cours d'une séance de la SMI, l’a révulsé » (p. 336).

1923 est une année cruciale pour notre compositeur, comme le souligne et le décrit en détail van Moere : c'est à cette époque qu'il rencontre Hélène Kahn-Casella (1876-1952), divorcée d'Alfredo Casella en 1919 et grande amie de Maurice Ravel. Celle-ci se battra bec et ongles pour faire reconnaître le jeune polonais dans la ville lumière, puisqu'elle voit en sa musique " un monde nouveau, une source nouvelle de beauté, et celle probablement dont j'avais le plus besoin, je le sens chaque jour "

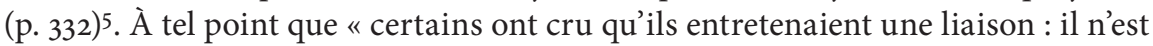
pas sûr que cela, pour des raisons différentes, ne les ait pas arrangés tous les deux " (p. 332). Mais cette admiration et cette dévotion de la pianiste française deviendront rapidement étouffantes pour Szymanowski. Du coup, il est déchiré entre l'agacement et la reconnaissance : « La Casella m'a naturellement rendu visite tous les jours, la pauvrette. Elle est ennuyeuse, mais c'est vraiment un fidèle petit bout de femme et elle est très dévouée » (p. 360) ${ }^{6}$.

Le Roi Roger op. 46 (1918-1924) répond, selon van Moere, à la question du renouvellement de l'opéra posée par ses contemporains : «Les bacchanales ? Elles n'ont plus rien à voir avec le traditionnel ballet d'opéra et correspondent à des moments essentiels de l'action intérieure, où non seulement les corps, mais aussi les consciences, engoncées au premier acte dans le hiératisme pesant du rituel byzantin, se libèrent enfin » (p. 392).

Dans les neuvième et dixième chapitres, l'auteur raconte l'épisode tourmenté qu'a été, suite au retour de Szymanowski en Pologne en 1927, la direction du Conservatoire de Varsovie de 1927 à 1932. Plusieurs de ses subalternes, dont le critique Piotr Rytel (1880-1928), lui mettront constamment des bâtons dans les roues, critiquant ses moindres faits et gestes. Il entreprend une véritable réforme du Conservatoire, tant du côté du corps professoral, qu'il souhaite rajeunir, que de l'enseignement musical. Szymanowski sera, dans ces années, très anxieux, au point de développer de graves troubles de santé. Dorothy Jordan-Robinson, la dédicataire du Roi Roger, ira même jusqu'à promettre à notre musicien deux mille dollars par an pendant dix ans, à condition qu'il quitte le Conservatoire et se consacre exclusivement à la composition (p. 461).

Les chapitres IX et $\mathrm{X}$ sont consacrés aux dernières années de Szymanowski en tant que directeur du Conservatoire et de ses derniers séjours à Paris, où il retourne de façon épisodique. Le compositeur traverse toujours une période difficile tant aux

5 Cité par l'auteur et tiré d'une lettre d'Hélène Kahn-Casella à Szymanowski du 15 décembre 1923.

6 Cité par van Moere et tiré d’une lettre à Kochanski du 14 août 1923. 
niveaux personnel, artistique, que financier. Szymanowski s'éteint le 29 mars 1937, des suites de la tuberculose; la Pologne lui réserve des funérailles officielles.

Les onze chapitres de ce volume sont suivis d'un catalogue complet et très détaillé des œuvres de Szymanowski, classées par numéros d'opus et faisant mention des noms de dédicataires, des maisons où les œuvres ont été éditées (ou précise si elles sont inédites), ainsi que de la date et du lieu de leur création.

Un sujet prédomine particulièrement dans cette biographie : celui du nationalisme musical, que l'auteur définit en se fondant entre autres sur les travaux de quelques spécialistes, notamment Daniel Beauvois (1995) et Ludwik Erhardt (1974). On retrouve dans la définition que donne Szymanowski de ce nationalisme, plusieurs traits auxquels d'autres compositeurs européens, contemporains du compositeur, se réfèrent. C'est le cas d'Alfredo Casella en Italie avec lequel Szymanowski partage une vision d'un nationalisme menant à l'universel et se fondant non pas sur le folklore de la nation, mais sur sa grande tradition musicale du passé.

Szymanowski réclame une renaissance de la musique polonaise. La démarche de notre compositeur, comme le précise l'auteur, est celle de la « quête d'une musique spirituellement ancrée dans l'esprit d'un peuple ou d'une race, dépassant les critères de la musique nationale telle que l'a conçue le XIX ${ }^{\mathrm{e}}$ siècle et que la conçoivent encore certains de ses plus farouches adversaires » (p. 404). Pour lui, le caractère national en musique ne réside pas tant dans des citations provenant du folklore, le meilleur exemple étant Chopin.

Szymanowski fait également une distinction entre la " nationalisation » de la musique et le «nationalisme » musical, suite à quoi il « dénonce un esperanto sans couleur ni caractère; l'art exprime d'abord une transcendance tout à fait compatible avec les particularités individuelles et nationales » (p. 563)7. Pour notre musicien, la musique polonaise ne doit pas se limiter au "fantôme figé d'une musique polonaise ou d'une mazurka » (p. 524). Il lancera cette affirmation dans de nombreux articles publiés notamment dans La Revue musicale en France et dans Muzyca en Pologne.

De manière générale, cette biographie de Karol Szymanowski est des plus intéressantes car elle comble un vide et contribue à mieux nous faire connaître ce compositeur trop souvent laissé pour compte. De plus, le sujet, très rarement traité, est pertinent pour quiconque s'intéresse à la musique du début du $\mathrm{XX}^{\mathrm{e}}$ siècle, puisqu'il montre la participation de Szymanowski à la vie musicale polonaise et française en plus de mettre l'accent sur ses œuvres à découvrir. La grande qualité de l'écriture et du langage utilisé par Didier van Moere facilite la lecture du récit de la vie de Karol Szymanowski. Par ailleurs, les analyses très précises et détaillées, par leur insertion dans le contexte de l'époque et de la vie de Szymanowski, facilitent une meilleure compréhension de l'œuvre.

\section{RÉFÉRENCES}

Beauvois, Daniel. 1995. Histoire de la Pologne. Paris : Hetier. Buch, Esteban. 2006. Le cas Schönberg. Paris: Gallimard

7 Cité par van Moere et tiré de l’article de Karol Szymanowski publié en 1933, "L'avenir de la culture». 
Cadrin, Paul. 2004. "Karol Szymanowski and Richard Strauss : Of Diverging Epiphanies ». Revue de musique des universités canadiennes 24, no $2:$ 29-43. Duchesneau, Michel. 1997. L'avant-garde musicale à Paris de 1871 à 1939. Sprimont : Mardaga.

Erhardt, Ludwick. 1974. La musique en Pologne. Paris : Fayard.

Palmer, Christopher. 1987. Szymanowski : Un compositeur à la croisée des chemins, essai traduit de l'anglais par Myriam Tcamitchian-Faure, avec la participation d'Alain Artaud. Arles : Actes Sud.

Samson, Jim. The Music of Szymanowski. London : Kahn and Averill, 1990.

__ [ [2008]. «Szymanowski, Karol». Grove Music Online [en ligne] <http:// www.oxfordmusiconline.com/subscriber/article/grove/music/27328?q=Ka rol+Szymanowski\&search=quick\&pos=1\&_start=1\#firsthit $>$ (consulté le 7 janvier 2009).

Justine Comtois

Adrian Thomas. 2005. Polish Music since Szymanowski. Cambridge, UK: Cambridge University Press. 384 pp. ISBN-13: 978-052-105472-o (paper).

Whether because of language barriers, political divisions throughout history, the lack of readily available materials, or a perceived distance from a "musicological mainstream," the majority of English-speaking music scholars have shied away from studying the artists and repertoires of East Central Europe. In most general histories of modernism, the region is all but ignored in favour of Vienna, Darmstadt, or New York. Richard Taruskin, writing on nationalism and historiography in 1996, famously problematized the "numerus clausus" quota system in the Western canon that draws a sharp line between Janáček and Bartók (among others) and grants a higher status to the latter, on the basis of an imaginary tally of influences on later modernist composers. Indeed, most master narratives of music history disallow close synchronic readings of local musical communities east of the Austro-German lands, such that only a lucky few individuals work their way into the narrative at all-sometimes as lone representatives of an exotic other, and always despite the limitations of their allegedly "peripheral" roots.

Thankfully, finding a substantive account of a musical community from this region has become a less rare event, where the scholarship seeks to gain exposure for the artists and their works, not to judge their apparent worth to subsequent historians, but to document musical events as they occurred. Such a work is Adrian Thomas's Polish Music since Szymanowski, a milestone of scholarship that balances the known and unknown. In this monograph, Thomas presents composers as familiar as Lutosławski and Penderecki alongside Palester, Szabelski, Moszumańska-Nazar, and a host of names barely encountered even in contemporary Poland, all of whom gave lifelong contributions to Polish musical life in the late twentieth century. While international acceptance is certainly acknowledged as laudable, it is not a prerequisite for inclusion 